\title{
El trabajador con problemas de salud mental. Pautas generales de detección, intervención y prevención
}

\author{
Workers with mental health problems. General guidelines for detection, interven- \\ tion and prevention \\ José Carlos Mingote Adán, Pablo del Pino Cuadrado, Raquel Sánchez Alaejos, Macarena Gálvez Herrer
y $M^{a}$ Dolores Gutiérrez García
}

Programa de Atención Integral al profesional sanitario enfermo (UVOPSE_PAIPSE). Consejería de Sanidad. Comunidad Autónoma de Madrid. Madrid. España.

\author{
Correspondencia: \\ José Carlos Mingote Adán \\ UVOPSE-PAIPSE \\ Pabellón 8, Ciudad Universitaria \\ 28040 Madrid. España \\ Tfno.: 913303926 \\ E-mail: jmingote.hdoc@salud.madrid.org
}

Resumen

Numerosos estudios han demostrado las relaciones entre condiciones psicosociales del trabajo y la salud mental de los empleados, y especialmente cómo la combinación de bajo control percibido y altas demandas laborales predicen determinados problemas de salud mental. Trastornos mentales tales como los de ansiedad y depresión tienen un efecto muy negativo sobre la calidad de vida y la capacidad funcional en el trabajo. Además, la salud mental del trabajador puede afectar a la percepción de las características del trabajo. Como consecuencia, es fundamental diseñar trabajos saludables, programas de reducción de estrés para los empleados y para la reincorporación laboral de empleados con trastornos mentales.

El objetivo del presente artículo es revisar las principales líneas de prevención, detección e intervención en las organizaciones, que pueden promover políticas saludables para la atención e integración de los trabajadores con trastorno mental.

Palabras claves: salud mental laboral, diagnóstico, prevención, condiciones psicosociales laborales.

Abstract

Numerous studies have demonstrated the relationship between psychosocial work conditions and the mental health of employees, and especially the combination of low perceived control and high labor demands predicts mental health problems. Mental health disorders such us anxiety or depression have a big detrimental effect on the individual's quality of life and the ability to function in the workplace. Mental health may also affect the perception of work characteristics. As a consequence, it is essential to design healthy jobs, stress reduction programs and return to work programs for employees with mental disorders.

The aim of this paper is to review the main lines of prevention, detection and intervention in organizations that can promote healthy policies for the care and integration of workers with mental disorder.

Key words: labour mental bealth, diagnosis, prevention, psychosocial work conditions. 


\section{INTRODUCCIÓN}

La prevención, detección y tratamiento de los problemas de salud mental en el ámbito laboral no es tarea sencilla debido a su carácter multidimensional, interviniendo factores personales, organizacionales y socioculturales. A esta complejidad se une el estigma que pesa sobre la enfermedad mental, causante, entre otros factores, de que menos de una tercera parte de las personas con trastornos mentales (en la población general) reciba asistencia sanitaria ${ }^{1-2}$. El abordaje de estos aspectos requiere por tanto una perspectiva multidisciplinar, con aportaciones de la medicina del trabajo, la medicina de familia y comunitaria, la psiquiatría, la psicología, la sociología, la enfermería, el trabajo social...

El ámbito de la Salud Mental Laboral requerirá por otro lado, de una estrecha colaboración entre los diferentes servicios asistenciales implicados (atención primaria, especializada, empresa), de forma que se permita optimizar los recursos sanitarios disponibles y dar una respuesta más eficaz a la demanda asistencial de los pacientes con trastornos mentales. Objetivos recogidos por Otero $(1995)^{3}$ para Atención Primaria, serían válidos para este abordaje multidisciplinar:

- Evaluación de factores psicosociales de riesgo.

- Identificación y orientación precoz de personas y grupos de riesgo.

- Diagnóstico y tratamiento de personas con trastornos mentales leves-moderados.

- Derivación de pacientes graves a nivel especializado.

- Actividades preventivas y de promoción de la salud mental en el ámbito sociolaboral, como se considera necesario para la detección precoz de la depresión mayor, el suicidio y el consumo de alcohol y otras drogas.

Como en otros ámbitos asistenciales ${ }^{4}$, será oportuno desarrollar distintas actividades protocolizadas como son:

- El Establecimiento de mecanismos de derivación y seguimiento compartido de casos.

- Sesiones de interconsulta de casos.

- Sesiones clínicas y teóricas.

- Grupos de aprendizaje, tipo Balint, etc. para lograr optimizar la colaboración entre los profesionales de este nuevo ámbito de conocimiento.

Para realizar este abordaje, quizás la primera dificultad a salvar sea la propia definición de "trastorno mental". No existe una definición completamente consensuada que especifique de forma adecuada los límites del concepto, aunque este término se utiliza para distinguir entre trastornos mentales y otras enfermedades médicas (definidas por la existencia de patología estructural, desviación fisiológica y/o etiología conocida).

En general, según el DSM-IV-TR ${ }^{5}$, el trastorno mental es conceptualizado como un síndrome o un patrón de comportamiento de significación clínica, asociado a un malestar presente, a una discapacidad funcional significativa o a un riesgo aumentado de sufrir un daño a la salud, de morir, de discapacidad o de pérdida de libertad.

A pesar de su complejidad diagnóstica, en los últimos años se han producido importantes avances en la validación de los diagnósticos psiquiátricos no sólo por criterios internos, tales como la consistencia de los síntomas y signos psicopatológicos, sino también por criterios externos que incluyen la realización de análisis de laboratorio, estudios genéticos, familiares y de neuroimagen, y el curso evolutivo de la enfermedad, que permiten delimitarla de otras enfermedades. Estas investigaciones han demostrado que gran parte de los trastornos mentales pueden ser diagnosticados y tratados con tanta eficacia como lo son otras enfermedades médicas, lo que permitirá a estos pacientes una mayor participación en la vida familiar y sociolaboral ${ }^{6}$. 
El grado de salud mental, inseparable de la salud general, se manifiesta en todos los ámbitos de la vida personal, incluido el trabajo, que a su vez la codeterminan de forma circular, positiva o negativamente ${ }^{7-9}$. Cuando pensamos en el trastorno mental dentro del ámbito del trabajo, hay tres efectos básicos que enlazan las interacciones complejas que tienen lugar entre empleo y salud mental:

1. Los efectos de las condiciones de trabajo (o desempleo) sobre la salud mental

2. Los efectos de la enfermedad mental sobre el desempeño laboral

3. Los efectos de otros factores como son variables individuales, estatus marital, hijos en el hogar, origen sociocultural, etc., que deben ser considerados simultáneamente.

En general, el hecho de tener un empleo protege la salud mental de hombres y mujeres. Incluso los pacientes con esquizofrenia que pueden mantener el empleo, se observa menor nivel de ansiedad y mayor nivel de iniciativa y actividad. Sin embargo, el trabajo puede también intervenir como causa o agravante de los problemas de salud mental a través de tres factores fundamentales: condiciones del medio ambiente material, medio ambiente psicológico y medio ambiente socioeconómico, que suelen actuar de forma acumulativa y negativa sobre la salud del trabajador.

La Ley de Prevención de Riesgos Laborales ${ }^{10}$ define las condiciones de trabajo como: "cualquier característica del mismo que pueda tener una influencia significativa en la generación de riesgos para la seguridad y salud del trabajador". Dentro de dichas condiciones, son habitualmente los factores psicosociales del trabajo los que determinan la presencia o no de posibles riesgos psicosociales y por tanto, los que se relacionan en mayor medida con posibles problemas de salud mental.

Estos factores psicosociales comprenden aspectos del puesto y del entorno de trabajo, como el clima y la cultura organizacional, las funciones laborales, las relaciones interpersonales en el trabajo (como el grado de apoyo/conflicto social), y el diseño y el contenido de las tareas, como el grado de autonomía y control en su ejecución. Los factores psicosociales de riesgo son los antecedentes, los estresores, los agentes etiológicos capaces de provocar estrés en los trabajadores, como son los diferentes aspectos de la organización laboral, cuando no se ajustan a las necesidades, expectativas y capacidades del trabajador, moderados por factores individuales y del contexto organizativo.

En la mayor parte de los estudios realizados sobre estos temas, se pone de manifiesto que los factores organizacionales de riesgo explican una parte importante de la varianza de las diferentes variables de riesgo analizadas, por encima de la explicada por las variables individuales. Estos datos señalan la importancia de prevenir en el origen y en la mejora de la calidad de las condiciones de trabajo, resultados que apoyan el desarrollo de programas de mejora de la calidad laboral, como a través de promover el entrenamiento en habilidades de comunicación y control de las demandas laborales.

Sin embargo, una perspectiva integradora deberá incluir también la consideración de los factores de vulnerabilidad y de protección individuales con programas de prevención secundaria que desarrollen competencias y habilidades en los trabajadores para el manejo y control de los estresores y minimización de sus efectos para la salud. El concepto de ajuste persona-ambiente es un marco teórico muy útil para estudiar la conducta humana en las organizaciones y para desarrollar programas específicos para la mejora de la salud del empleado, así como para la prevención y control del estrés laboral mediante la aplicación de medidas correctoras, promoción de mecanismos compensadores o estructuras psicosociales de apoyo que permitan el empleo de estrategias adaptativas para el afrontamiento de los riesgos psicosociales del trabajo.

En otros artículos del presente monográfico se afronta de forma específica la influencia de determinados factores psicosociales del trabajo, riesgos psicosociales o condiciones del trabajador que se relacionan con su salud psíquica y laboral. El objetivo del presente artículo es reflexionar sobre algunas pautas de actuación ante trabajadores con problemas de salud mental, revisando algunos aspectos esenciales sobre su detección y diagnóstico, intervención y posibles líneas de prevención en el entorno laboral. 


\section{DETECCIÓN Y DIAGNÓSTICO DE LOS TRASTORNOS MENTALES EN EL LUGAR DE TRABAJO}

\section{Detección de los problemas de salud mental}

Cada trabajador tiene unas actitudes diferentes en función del grado de salud, competencias, expectativas, valores y motivaciones, que van a condicionar su grado de satisfacción/insatisfacción laboral y vital, el nivel de estrés y de calidad de vida, tanto personal como laboral ${ }^{11-12}$.

Las personas con cualquier trastorno mental pueden experimentar varias alteraciones del comportamiento que constituyen signos de alerta en el ámbito laboral indicativos de la necesidad de una evaluación inicial.

Así por ejemplo la Guía de Salud del MIR editada por la Organización Médica Colegial y la Fundación Galatea ${ }^{13}$, destaca los siguientes signos de alarma (que en general, son aplicables a múltiples profesiones): absentismo laboral, desmotivación y disminución en el rendimiento laboral, desobediencia e incumplimiento de las tareas propias del rol laboral, conflictos interpersonales, irritabilidad excesiva y conductas agresivas en el trabajo, falta de colaboración y evitación del contacto con los compañeros y dificultad en la toma de decisiones.

Estos signos de alarma son similares a los que sugieren otras organizaciones profesionales $^{14}$ :

- Cambios en la personalidad: expresiones de distrés, tristeza, ansiedad, ira, hostilidad, expresiones de desvalorización o desesperanza constantes.

- Cambios de conducta: deterioro de la calidad del trabajo, cambio negativo en el rendimiento, ausencias o retrasos repetidos en actividades importantes, búsqueda continúa de ventajas especiales, frecuentes conflictos interpersonales.

- Cambios físicos: deterioro del aspecto físico y del autocuidado, cambios importantes de peso, fatiga excesiva, alteraciones del sueño, indicadores físicos de adicción (marcas, edor...).

- Otros cambios: quejas de usuarios y/o compañeros, ordenes inapropiadas, ausencias no justificadas, conflictiva extra-laboral paralela a la del trabajo, expresiones de preocupación por parte de compañeros y/o supervisores.

La decisión de consultar a un especialista depende prioritariamente del propio trabajador. Este factor será esencial para tomar una adecuada conciencia de enfermedad, para comprometerse con su tratamiento, para adquirir un sentimiento de autocuidado y autoeficacia respecto a la propia salud, y por todo ello, para el éxito terapéutico. La primera y mejor ayuda desde la red de apoyo (sociofamiliar y laboral) consistirá en ayudarle a detectar dicha necesidad y fomentar su autonomía en la petición de ayuda.

En otras ocasiones, es el médico de atención primaria, quién detecta la posible problemática y decide tratar y/o derivar al paciente a salud mental (sea de forma ambulatoria o bien hospitalaria en los casos graves). Merece la pena recordar en este punto, que según algunos estudios en el ámbito europeo, el porcentaje de morbilidad psiquiátrica oculta oscila entre el 40 y el $65 \%$ de casos en población general ${ }^{15-16}$.

El ámbito laboral es también un lugar frecuente de detección de casos. En muchas ocasiones la consulta de los Servicios de Prevención de Riesgos Laborales es el espacio donde el problema laboral y el de salud mental se presentan de forma conjunta.

Así, Atención Primaria, Salud Mental y Salud Laboral pueden constituir una red de apoyo profesional eficaz que haga posible una atención integral de calidad centrada en la comunicación con el paciente, agente principal y responsable del cuidado de su propia salud. Para hacer factible este modelo orientado a la prevención y la promoción de la salud se requiere el desarrollo de mecanismos eficaces de coordinación interdisciplinar 
en una red de apoyo sociosanitario profesional centrada en la comunicación con el paciente. Esta coordinación será especialmente relevante si se trata de:

- Trabajador con trastorno mental crónico

- Caso clínico complejo (por ejemplo con patología dual, complicaciones psicosomáticas, etc.).

- Empleos de elevada responsabilidad sobre terceros (profesiones asistenciales o de alto riesgo físico para sí mismos o terceras personas, por ejemplo).

Los trastornos psiquiátricos más frecuentemente observados en el ámbito laboral son los mismos que con mayor frecuencia se detectan en la población general ${ }^{17}$ :

\section{Trastornos afectivos (principalmente depresión y ansiedad, incluyendo el tras- torno por estrés postraumático)}

Según algunos estudios realizados en empresas españolas ${ }^{18}$, la prevalencia global de trastornos afectivos durante el año 2001 fue de un 15,8\% ( 8,4\%-24\%). Por ocupación, las prevalencias más altas las presentaron el personal administrativo (18\%-26,7\%), los operarios de maquinaria (21\%) y los trabajadores no cualificados $(21,9 \%)$.

Cuando existe una recurrencia depresiva, ésta se acompaña de un rápido empeoramiento del rendimiento sociolaboral, referido tanto a la eficacia en la realización de las tareas como a la aparición de conductas de aislamiento y frecuentes conflictos interpersonales. En el trabajo, las personas con depresión ${ }^{19}$ :

- Están desmotivadas en mayor medida.

- Mantienen deseos de abandono profesional o retirada prematura.

- Establecen peores relaciones personales con problemas de comunicación.

- Se ven implicadas con mayor frecuencia en situaciones de conflicto laboral.

- Disminuyen su rendimiento, capacidad de concentración y eficacia.

En el ámbito psicosocial, la depresión produce un gran sufrimiento en la persona afectada y en las personas de su entorno, con importantes repercusiones laborales y socioeconómicas, debido a la pérdida de productividad, al absentismo y a las posibles bajas laborales relacionadas.

Una reciente revisión bibliográfica sobre depresión y trabajo ${ }^{20}$ señala como los predictores de la depresión son multifactoriales y señalan por ejemplo:

- Diferencias de género: en mujeres, el bajo nivel de autoridad, los trabajos de baja cualificación del área industrial o los trabajos que tienen alta demanda emocional, están asociados con el riesgo de depresión, mientras que en hombres son el estrés, la inseguridad laboral, los trabajos pasivos y trabajos tanto de alta como de baja cualificación, los más frecuentemente asociados con depresión.

- En el ámbito del trabajo: la inseguridad laboral, el bajo apoyo social, la baja remuneración económica, el pobre clima laboral, el desequilibrio entre la tensión laboral y la recompensa al esfuerzo, el bajo control laboral son condiciones relacionadas estrechamente con mayores índices de depresión.

El Consejo de Administración de la OIT aprobó el 25 de marzo de 2010 una nueva lista de enfermedades profesionales, en la que se incluyen por primera vez los trastornos mentales y del comportamiento (epígrafe 2.4). Concretamente, el punto 2.4.1., codifica al Trastorno de estrés postraumático (TEPT) tras exposición a un estresor traumático en el trabajo, como puede ocurrir tras accidentes graves y a consecuencia de experiencias de violencia grave, externa o interna que ponen en peligro la vida o la integridad del trabajador.

Se trata del único trastorno mental definido por su etiología específica en la nosología actual. Es uno de los trastornos de ansiedad más frecuentes en la población general, aunque se reconoce que está infradiagnosticado y que la mayoría de los pacientes que le padecen no reciben tratamiento específico adecuado. Este hecho se explica por varios 
factores: porque estos pacientes buscan ayuda por síntomas inespecíficos de malestar que sugieren otros trastornos mentales, por la evitación característica de los estímulos relacionados con la experiencia traumática y por un enfoque asistencial orientado a los síntomas y no al cuidado de la persona que los padece.

\section{Abuso y dependencia del alcohol y otras drogas}

Algunos trabajos internacionales ${ }^{21}$ concluyen que en torno al $20 \%$ de los empleados consumen bebidas alcohólicas y otras sustancias de abuso de forma excesiva e inapropiada, con un elevado coste por pérdida de productividad, siniestralidad laboral y numerosas enfermedades relacionadas con este motivo. En España, según los datos de la última encuesta del Plan Nacional sobre Drogas, aproximadamente un $10 \%$ de las personas activas son bebedoras de riesgo y el $27 \%$ ha consumido cannabis en el último mes, siendo éstas las dos drogas más prevalentes y con más repercusión en el ámbito laboral.

Son diversos los riesgos psicosociales que median en el incremento del riesgo para el consumo de drogas, como el estrés laboral y la turnicidad. Además, este tipo de adicciones generan problemas laborales tales como accidentes de trabajo con riesgo para si mismo y terceras personas, absentismo, recurrentes bajas laborales, bajo rendimiento, baja calidad del trabajo y conflictos interpersonales.

Se ha constatado la relación entre sobrecarga de trabajo, ambigüedad y conflicto de rol con un riesgo 27,5 veces más frecuente de abuso de alcohol. El desarrollo de múltiples roles incrementa las posibilidades de experimentar mayores niveles de demandas y de exposición a más situaciones estresantes, al conflicto familia-trabajo y a la búsqueda de estrategias evitativas de afrontamiento como pueden ser las adicciones ${ }^{22}$.

La identificación precoz del problema y la motivación para su tratamiento será un elemento clave para la prevención de los efectos personales y sociolaborales de la adicción.

\section{Pautas diagnósticas generales}

Los dos sistemas de clasificación de los trastornos mentales más utilizados, la Clasificación Internacional de Enfermedades (CIE-10 de la OMS, 1992) y el DSM-IV-TR (2000), utilizan criterios operativos de diagnóstico, es decir, con definiciones formales de las categorías diagnósticas, con criterios estrictos de inclusión, de exclusión, y con especificadores temporales, lo que ha incrementado de forma notable la fiabilidad diagnóstica y con ella su utilidad clínica.

Sin embargo, en ocasiones estas clasificaciones se alejan de la realidad de la clínicalaboral. Por ejemplo, en la Clasificación DSM-IV-TR, la especificación "Problema laboral" aparece entre otros problemas adicionales que pueden ser objeto de atención clínica en el Eje I y dentro de los Problemas psicosociales y ambientales en el Eje IV, donde aparecen solo algunos ejemplos como: "desempleo, amenaza de pérdida de empleo, trabajo estresante, etc.", sin un mayor desarrollo posterior. Tampoco en la Clasificación Internacional de Enfermedades (CIE) ocurre esto.

La evidencia científica avala que una práctica clínica de calidad ha de basarse en una medicina personalizada, es decir "la medicina de la persona como un todo" entendida como sujeto singular que tiene un conjunto de atributos, capacidades, problemas y experiencias, que es preciso tener en cuenta, y como miembro de un grupo que está sujeto a diferentes influencias familiares, sociolaborales y culturales. Incluye el estudio de la enfermedad (disease) y del padecimiento (illness), la percepción y experiencias subjetivas de los pacientes y cada uno de estos aspectos, debe ser evaluado de forma adecuada $^{23}$. Además, desde un punto de vista clínico los trastornos mentales podrán clasificarse según su evolución temporal en agudos o crónico-recurrentes, y según su gravedad clínica en leves-moderados o graves. 
El diagnóstico del paciente enfermo debe ser multidimensional y no reduccionista, con inclusión del síndrome clínico, las características del funcionamiento personal, en el ámbito familiar y sociolaboral, así como de los agentes etiológicos que lo hayan determinado, sean de tipo biológico y/o psicosocial. El diagnóstico debe incluir la dimensión evolutiva, la relacional (como conflictos y apoyo social), así como las percepciones y creencias del paciente acerca de su enfermedad.

El diagnóstico diferencial de los trastornos mentales relacionados con circunstancias estresantes laborales no puede depender de la mera descripción de los hechos y de la interpretación subjetiva del trabajador, aunque sea muy importante la percepción que éste tiene. Aceptar el carácter causal de un estresor supone también llevar a cabo una interpretación que no puede demostrarse con certeza, sino como probabilidad. Resultan de utilidad los siguientes aspectos prácticos a tener en cuenta ${ }^{24}$ :

1. Excluir la posibilidad de que el estresor sea un resultado del trastorno mental más que su causa.

2. Tener en cuenta que algunos pacientes se descompensan al estar sometidos a estrés porque padecen un trastorno de personalidad preexistente.

3. Tener en cuenta el diagnóstico de trastorno adaptativo en presencia de un estresante identificable al que siguen algunos síntomas psiquiátricos, una vez excluidos los trastornos mentales, clínicos y de la personalidad.

4. Para diagnosticar un trastorno adaptativo se requiere la presencia de un estresante identificable que desencadene los síntomas.

5. Considerar la posibilidad de que exista un problema psicosocial que no sea originado por un trastorno mental, sino por problemas laborales, familiares o conflictos interpersonales.

Desde el modelo interactivo diátesis-estrés, las consecuencias de los acontecimientos vitales estresantes pueden ser: normales, adaptativas o patológicas y desadaptativas. En cualquier caso, siempre se parte de la demanda que presenta el paciente, con sus quejas, síntomas y disfunciones, cómo interfieren en sus diferentes entornos vitales (incluido el laboral) y sus opiniones acerca de la causa del malestar que padece. En este sentido se deben escuchar con respeto las opiniones de los pacientes, pero será también importante indagar, junto a él, posibles factores de vulnerabilidad individual que participen del problema.

El objetivo de una completa evaluación será:

- La identificación de síntomas y problemas que nos ayude a establecer un diagnóstico

- Determinar los posibles objetivos de intervención (farmacológica, terapéutica, laboral y psicosocial)

- Seleccionar las técnicas de intervención más adecuadas en función de las circunstancias concretas del paciente y del contexto (intervención en el lugar de trabajo, cómo y con quién, posibles derivaciones, etc)

- Valorar los resultados de la intervención y registrar la evolución del paciente.

Para realizar un adecuado proceso diagnóstico es importante recordar algunos procedimientos básicos:

\section{La entrevista clínica}

Es el elemento básico de evaluación. Requiere construir una relación de confianza que proporcione seguridad y actitudes de empatía. La entrevista clínica orientada a la exploración de los síntomas y los factores etiológicos relacionados, constituye a veces una verdadera "prueba de esfuerzo" para el paciente y también para el profesional que le atiende. La valoración debe ser gradual y contenedora de la ansiedad que se reactiva en ese momento. El profesional puede observar, monitorizar y modular el estado emocional del paciente a lo largo de la entrevista, según el estado clínico y la evolución del paciente. 
Basadas en los sistemas vigentes de clasificación se han desarrollado varias entrevistas estructuradas para el diagnóstico (DIS; CIDI; SCAN), aunque su uso clínico ha sido escaso debido al consumo de tiempo que requieren y a la complejidad de algunas de ellas, que requieren un entrenamiento avanzado y una sólida formación psicopatológica.

La recogida de información se adaptará a cada caso concreto, pero en general incluirá información sobre la conducta, las actitudes y las emociones actuales y pasadas, además de un historial detallado de la vida del individuo en general y del problema que presenta.

El examen del estado mental abarcará:

1. Apariencia y conducta (vestimenta, higiene, expresión facial, conducta motriz...)

2. Procesos de pensamiento (de forma aproximada a través de la continuidad, contenido y velocidad del habla)

3. Estado de ánimo y afecto (estado emocional predominante, concordancia o no con lo expresado...)

4. Funcionamiento intelectual (tipo de vocabulario, uso de abstracciones o metáforas...)

5. Sensorio, conciencia general del entorno (en relación con la persona, el tiempo y el espacio)

Además de esas circunstancias de carácter clínico, en la evaluación dentro de un contexto laboral cobra también importancia la historia laboral del sujeto, adaptación durante la misma o posibles problemas previos.

A partir de esta información se dirigirá el resto de la entrevista clínica, ajustándose a cuestiones específicas en función del posible trastorno a evaluar, hábitos de consumo si es el caso, exámenes físicos complementarios, analíticas y otras pruebas diagnósticas.

\section{Uso de instrumentos y cuestionarios de evaluación}

La evaluación psicológica por medio de instrumentos de medición puede ser complementaria a otros métodos diagnósticos y de gran utilidad. Sin embargo, es importante no olvidar que todo instrumento tiene unos indicadores de fiabilidad y validez a tener en cuenta, que respecto a los cuestionarios, en su mayor parte, se trata de autoregistros, lo que implica que los resultados obedecen a la percepción subjetiva de la persona que los complementa, y que en su mayoría, no deben usarse como criterio único de diagnóstico.

Para mantener en lo posible la objetividad en la aplicación de un instrumento de evaluación y no añadir sesgos, se requiere una aplicación y corrección rigurosa (a todas las personas de igual manera, con instrucciones claras y siempre las mismas).

Además, la selección del instrumento debe estar debidamente justificada por su objetivo de evaluación y pertinencia para cada caso concreto. Algunos ejemplos instrumentos generales de utilidad en el ámbito clínico laboral son:

La Escala de Acontecimientos Vitales y Dificultades ${ }^{25}$ desarrollada por Brown y Harris que utilizando dicha escala concluyeron que el trabajo protege la salud mental (en una muestra de 395 mujeres trabajadoras), mientras que su falta constituye un significativo factor de vulnerabilidad para la depresión. Identificaron también el efecto protector de las relaciones de apoyo y cómo las personas experimentan simultáneamente varios tipos de estresores, pertenecientes a sus diferentes ámbitos vitales que interactúan entre sí de forma dinámica. Así, por ejemplo, el estrés laboral puede exacerbar la frecuencia e intensidad de los conflictos familiares, que, a su vez, sensibilizan al trabajador para los estresores laborales.

Se trata de una entrevista semiestructurada, con una lista de 38 tipos de acontecimientos, agrupados en 8 categorías. El entrevistador, tras una serie de preguntas, utiliza un manual que tipifica estrictamente qué tipo de incidente debe ser calificado 
como acontecimiento vital y cuáles resultan significativos. Es un instrumento muy completo pero difícil de utilizar en poblaciones amplias por la necesidad de entrevistadores entrenados y el tiempo de administración y corrección.

El Cuestionario de Salud General de Goldberg ${ }^{26}$ : El cuestionario inicial consistía en 60 preguntas. Posteriormente se han elaborado versiones más cortas de 30, 28 y 12 ítems respectivamente que han mostrado igual utilidad según diferentes estudios de validación efectuados ${ }^{27-29}$. Este cuestionario fue elaborado por Goldberg con la intención de identificar la severidad de problemas psiquiátricos menores, por lo cual se considera que, en realidad, lo que mide es salud mental más que el estado de salud general.

El SCL-90 de Derogatis et al..$^{30}$ es uno de los cuestionarios de autoinforme más utilizados internacionalmente para la evaluación de síntomas de psicopatología, Se ha empleado con éxito en clínica e investigación (evaluación de la eficacia del tratamiento, screening, separación entre grupos de pacientes, determinación del nivel de gravedad psicopatológica, etc.). Incluye nueve escalas que cubren la evaluación de nueve dominios psicopatológicos básicos (somatización, obsesión-compulsión, sensibilidad interpersonal, depresión, ansiedad, hostilidad, ansiedad fóbica, ideación paranoide, y psicoticismo). Davison et al. ${ }^{31}$ desarrollaron la versión abreviada de 45 ítems, el Symptom Assessment-45 Questionnaire $(S A-45)$, manteniendo las principales virtudes del SCL-90 y sus mismas dimensiones, y que ha sido adaptado y validado en población española por Sandín et al. ${ }^{32}$

\section{PAUTAS GENERALES DE INTERVENCIÓN CLÍNICO-LABORAL}

Autores como Zúñiga ${ }^{33}$ plantean un sistema de clasificación de los enfermos con trastornos mentales en función de otras variables distintas al diagnóstico, concretamente, en relación con variables de tipo psicosocial; de forma que analiza gravedad clínica, disfunción psicosocial, conductas de riesgo y comorbilidad médica, definiendo en función de todo ello el grado de carga asistencial y lugar de intervención:

1. ${ }^{\circ}$ Grupo de trastornos adaptativos, con sintomatología aguda y leve, sin disfunción psicosocial ni conductas de riesgo y que supone una carga asistencial baja.

2. ${ }^{\circ}$ Grupo ansioso-depresivo, con presencia de sintomatología leve-moderada crónica, escasa disfunción psicosocial, problemas laborales leves, sin conductas de riesgo ni complicaciones médicas. Presentan una gravedad moderada-baja y una carga asistencial media-baja. Estos dos primeros grupos de pacientes podrían ser tratados a nivel de atención primaria y por otros especialistas que no son de salud mental.

3. ${ }^{\circ}$ Grupo depresivo-psicótico, caracterizado por sintomatología moderada y riesgo de suicidio, problemas laborales y familiares moderados, según las demandas de su rol profesional, gravedad moderada y carga asistencial moderada, según el grado de adherencia terapéutica.

4..$^{\circ}$ Grupo inestable-conflictivo, con síntomas graves, problemas importantes de autocuidado, desempleo temporal, conflictos familiares, conductas de riesgo y complicaciones graves en su tratamiento, como oposición al mismo, vínculo de mala calidad con los profesionales que le atienden, gravedad alta y carga asistencial elevada.

5. ${ }^{\circ}$ Grupo de alta discapacidad laboral y social, graves problemas de autocuidado, desempleo crónico, síntomas positivos y negativos graves, complicaciones graves con el tratamiento con oposición al mismo y difícil vínculo con los profesionales sanitarios, gravedad elevada y carga asistencial muy alta.

Los pacientes de estos tres últimos grupos requieren el tratamiento coordinado por una amplia red de profesionales sanitarios en la que junto a los de salud mental deben incluirse los de otras especialidades sociosanitarias para proporcionarles el apoyo profesional que precisan par su tratamiento eficaz. 
Para ello será necesaria una adecuada coordinación y seguimiento aceptado por el paciente y por las demás partes intervinientes, de forma individualizada, con contrato terapéutico y las medidas que se consideren necesarias para lograr un control escrupuloso de la adherencia terapéutica de los pacientes con trastorno mental grave, según las demandas y responsabilidades específicas del rol profesional de cada paciente ${ }^{34}$.

Para poder reducir las consecuencias personales y sociales y los costes que acarrean los problemas de salud mental en las organizaciones será necesario:

- Un diagnóstico precoz e instauración de un tratamiento efectivo, para mantener la buena salud del paciente y prevenir la cronicidad y complicaciones graves.

- Prevención de nuevas recaídas: el objetivo clínico debe ser el tratamiento de la enfermedad y no sólo del episodio agudo, especialmente en trastornos como la depresión mayor.

- Habitualmente, la estrategia terapéutica más eficiente para la depresión crónica y recurrente es la combinación de farmacoterapia y psicoterapia. Ocurre lo mismo con la prevención de recaídas del trastorno bipolar, tras la introducción de varios anticonvulsivantes eutimizantes de nueva generación, igual que los clásicos carbonato de litio, ácido valproico y carbamazepina, después de observar la utilidad de abordajes terapéuticos integrados.

- Las elevadas comorbilidades relacionadas genéticamente con el episodio depresivo mayor (p. e. trastorno de angustia, trastorno obsesivo, bulimia nerviosa) implican un peor pronóstico, aunque suelen respondan a los mismos tratamientos, como los inhibidores selectivos de la recaptación de serotonina.

- En el caso de los episodios psicóticos, la detección precoz, el diagnóstico diferencial y el afrontamiento integral y multidisciplinario, permitirán un abordaje efectivo como ya expusimos en previos trabajos en esta revista ${ }^{35}$.

- Los programas de atención a trabajadores con adicciones tendrán como objetivo prioritario que el trabajador con problemas de alcohol y/u otras drogas recupere su salud y pueda volver a normalizar su vida laboral.

En todo ello será esencial el Servicio de Prevención de Riesgos Laborales ya que:

1. ${ }^{\circ}$ Determina la aptitud del empleado para el desempeño de su trabajo.

2. ${ }^{\circ}$ Detecta en numerosos casos los problemas de salud mental y posibles abusos de sustancias.

3. Pueden determinar la posible condición de "trabajador especialmente sensible", que incluye a los trabajadores que, por sus propias características personales o estado patológico conocido (incluidos aquellos que tengan reconocida la situación de discapacidad física, psíquica o sensorial) tienen aumentada su vulnerabilidad individual a los riesgos laborales.

4. ${ }^{\circ}$ En colaboración con otros servicios, puede gestionar y realizar un seguimiento del tratamiento rehabilitador para los empleados que lo precisen.

Se considera necesario protocolizar la derivación de pacientes a los servicios especializados de salud mental, según criterios operativos, como los siguientes propuestos por Ciurana y Tizón ${ }^{36}$ :

- Pacientes que presentan un trastorno depresivo mayor grave.

- Pacientes con antecedentes personales y/o familiares de suicidio, ante situaciones de riesgo.

- Cuando el paciente realiza conductas que suponen un riesgo real para él mismo o para los demás, como en el ejercicio de su profesión.

- Pacientes con el diagnóstico de psicosis o con síntomas psicóticos que requieren un diagnóstico preciso.

- Ante conductas disfuncionales con trastornos graves de la personalidad. 
- En general, cuando el médico del Servicio de prevención de Riesgos Laborales considere que precisa el asesoramiento y la ayuda diagnóstica o terapéutica del equipo de salud mental.

En todos los casos es prioritaria la preparación adecuada del paciente antes de realizar la derivación, incluso a través de varias visitas, aunque en otros casos se precisa hacerlo de forma urgente e involuntaria, siempre que la indicación esté justificada desde el punto de vista clínico y de la dinámica asistencial preestablecida.

\section{PREVENCIÓN DE LOS PROBLEMAS DE SALUD MENTAL EN EL TRABAJO Y PROMOCIÓN DE LA SALUD}

Prevención en salud mental implica plantear pautas de actuación para reducir la incidencia, prevalencia y recurrencia de los trastornos mentales, evitando, retrasando o disminuyendo, el impacto de la enfermedad en la persona afectada, su familias y la sociedad.

El modelo de prevención de la enfermedad incluye tres perspectivas complementarias, la prevención primaria, secundaria y terciaria (o intervención propiamente dicha):

\section{La Prevención Primaria}

Está orientada a reducir la incidencia de los problemas de salud a través del conocimiento y eliminación de los factores causales en las personas o grupos de riesgo, por medio de programas de educación sanitaria para mejorar la calidad de vida laboral y el afrontamiento de los factores de riesgo para la salud.

En el caso de la salud laboral, la línea de actuación prioritaria se centrará en la potenciación de las políticas de prevención de riesgos laborales, la actuación sobre los factores organizacionales, el ajuste de expectativas en los profesionales y la potenciación de factores motivadores (formación continuada, carrera profesional).

Una medida fundamental será la educación sanitaria para el conocimiento y control de los factores de riesgo conocidos. A su vez, será necesario aumentar los recursos para la protección de la salud y la mejora de la calidad de vida laboral mediante estrategias de intervención dirigidas a las condiciones y los procesos de trabajo optimizando la gestión de los recursos humanos a través de:

a) Enriquecimiento del trabajo incrementando la autonomía del trabajador, la calidad de la supervisión y la formación continuada.

b) Favoreciendo estilos de supervisión y de liderazgo participativo-democráticos.

c) Desarrollo de la carrera profesional según criterios objetivos y evaluables de calidad científica y de justicia.

Lamentablemente no es posible evitar todos los factores estresantes, pero a eso se une el hecho de que, en ocasiones, existen otros estresores autogenerados por el propio paciente, debido a características de personalidad ${ }^{37}$, estrategias poco funcionales de afrontamiento o vulnerabilidad genética a algún trastorno mentales (como puede ocurrir en la depresión).

Las estrategias organizacionales que favorezcan la integración laboral y el buen clima de trabajo serán determinantes para incrementar la autoeficacia percibida, un autoconcepto positivo y una buena integración social del individuo. Esto será especialmente importante en el caso de trabajadores con mayor vulnerabilidad a la depresión, que pueden sobre-implicarse en el trabajo para intentar mejorar su mala autoestima básica y neutralizar los sentimientos negativos de incapacidad y desvalorización personal, Cuando la depresión se agrava se produce un cambio de actitud, incrementándose la irritabilidad y por tanto la conflictividad, disminuyendo la involucración con el trabajo e incrementándose los deseos de abandono y las conductas de absentismo. 
Igualmente, se ha constatado la importancia del deterioro en los roles sociolaborales y en las relaciones interpersonales como consecuencia de la depresión, evaluando esta situación tanto de forma objetiva como subjetiva o percibida. En este último caso, destaca la insatisfacción y malestar experimentado en el trabajo y cómo, por el contrario, cuando la depresión mejora, se incrementa la satisfacción e incluso el rendimiento laboral de forma paralela a la disminución de los síntomas positivos.

\section{La Prevención Secundaria}

Se orienta al diagnóstico precoz y al tratamiento más eficaz para reducir la duración del trastorno y el riesgo de complicaciones.

En el terreno laboral, la detección temprana de los casos con mayor riesgo, así como la intervención tanto a nivel individual como grupal (equipos de trabajo), dotando a los profesionales de herramientas de afrontamiento y competencias de manejo y control de los riesgos, serán pautas de actuación esenciales.

Para el diagnóstico precoz de los problemas de salud mental, se requiere de la colaboración de todos los profesionales de los Servicios de Prevención de Riesgos Laborales y de Salud Mental. Se recomienda un modelo de asistencia conjunta y combinada, en el que los profesionales sanitarios de los distintos estamentos y especialidades implicados en el caso trabajen en equipo, cada uno en su propio contexto, bajo la dirección del responsable del caso. Un aspecto decisivo en la prevención de recaídas consistirá en ofrecer la estructura adecuada para el control y la mejora de la adherencia terapéutica. Los familiares y compañeros son indispensables para ello, hacen la función de observadores y personas que dan apoyo, proporcionan feedback al clínico sobre la recurrencia precoz de los síntomas, y reiteran al paciente la importancia de acudir a las visitas y tomar la medicación.

Buscar las claves diagnósticas en el comportamiento de la persona afectada y síntomas de los que nos informa, realizar la exploración del estado mental actual y evaluar los criterios diagnósticos de los posibles síndromes que puede padecer, realizar una historia psiquiátrica (antecedentes personales y familiares, curso e intensidad del trastorno, impacto en la vida del paciente, enfermedades médicas y consumo de sustancias de abuso), realizar una formulación diagnóstica multiaxial y formular un pronóstico ${ }^{38}$.

Según la información científica actualmente disponible, es prioritario partir del conocimiento de las diferentes trayectorias o mecanismos etiopatogénicos posibles para padecer un trastorno mental, ya que eso hará factible promover intervenciones preventivas eficaces $^{39-42}$ :

\section{Exposición acumulativa a un riesgo psicosocial del trabajo}

Por ejemplo, ante el estrés laboral percibido por falta de control sobre el entorno o la tarea y ausencia de oportunidades para utilizar las propias capacidades, el rendimiento disminuye y se origina un estado de fatiga que puede correlacionar con trastornos mentales, como los adaptativos, así como con enfermedades psicosomáticas relacionadas con el estrés como las cardio y cerebro-vasculares, dolores osteomusculares, etc.

Los factores estresantes laborales comunes (no traumáticos), pueden causar un trastorno adaptativo o reacción desadaptativa relacionada con el trabajo. Se distinguen cinco posibles reacciones desadaptativas en el DSM-IV: con ansiedad, con estado de ánimo depresivo, con trastorno de comportamiento, con alteración mixta de las emociones y el comportamiento, y con estado de ánimo mixto, ansioso y depresivo, con posibilidad de que se mezclen varias de estas reacciones. La reacción desadaptativa ha de ocurrir en un intervalo de 3 meses desde la aparición del estresor y no persistir más de 6 meses, salvo que se trate de un estresor crónico.

Diversos estudios han sugerido la existencia de una asociación entre elevado estrés laboral y un mayor riesgo de depresión, aunque esté condicionada por los sesgos 
subjetivos de los empleados. Recientemente se ha constatado una relación significativa entre altas demandas laborales tasadas por un experto externo y un riesgo aumentado de depresión mayor ${ }^{43}$. Los resultados de este estudio apoyan la necesidad de realizar intervenciones preventivas para el diseño de empleos saludables y programas de reducción de estrés basados en el rediseño de trabajos con menores demandas, mayores control del trabajador, a través de mayores posibilidades de cooperación, comunicación y aprendizaje.

Cuando el estrés ha causado mella en la salud mental, a las medidas organizacionales y de desarrollo de competencias y habilidades en el trabajador mencionadas debe unirse un trabajo psicoterapéutico sobre las vulnerabilidades individuales para el manejo del estresor y en la elaboración del daño. Esta acción conjunta: trabajo sobre estresores objetivos del trabajo, recursos y vulnerabilidades personales, conseguirá ayudar al profesional a manejar esa crisis pero también le aportará competencias para futuros acontecimientos problema.

\section{Exposición a riesgos psicosociales del trabajo que contribuyen al desencade- namiento de un trastorno mental para el que el trabajador es vulnerable}

Aquí el riesgo psicosocial del trabajo interviene como factor desencadenante o agravante. Ocurre por ejemplo en casos de trastornos depresivos y de ansiedad, sobre todo en los primeros episodios. Se atribuye a las situaciones estresantes el 10-15\% de la varianza sobre la sintomatología depresivo-ansiosa que padece el paciente. Se trata de trastornos de etiología multifactorial, de forma que cada factor explica una proporción relativamente pequeña de la varianza, lo que obliga a tener en cuenta diferentes factores de vulnerabilidad o modificadores, genéticos, evolutivos (como carencia afectiva infantil) psicológicos (como estilo atribucional negativo y baja autoestima) y sociales (como la falta de apoyo social).

\section{Exposición a situaciones laborales traumáticas}

Como puede ocurrir en casos de accidentes laborales graves, haber sido víctima de un acto violento en el trabajo, y en algunas ocasiones, tras situaciones crónicas y graves de acoso psicológico en el trabajo.

Estos riesgos se relacionan con alteraciones a la salud como el trastorno de estrés postraumático. Cuando esto ocurre, el que la intervención sea preventiva o terapéutica dependerá en gran parte del momento en que se produzca y del caso clínico concreto. En líneas generales, será importante ayudar al trabajador a establecer la conexión entre el acontecimiento traumático y su sintomatología, la "normalización" de las reacciones de miedo y confusión, y a procesar emocional y cognitivamente lo acontecido.

\section{Padecer un trastorno mental puede relacionarse con una menor tolerancia posterior a situaciones estresantes}

Este hecho será necesario tenerlo en cuenta y evaluarlo, especialmente para establecer las pautas adecuadas de la reincorporación laboral.

\section{La Prevención Terciaria (o Intervención)}

Se centra, como hemos visto en el apartado anterior de "Pautas generales de intervención clínico laboral", en intervenir sobre el problema de salud mental y disminuir el riesgo de las recaídas y complicaciones ulteriores.

Un ejemplo aplicado de esta actuación lo constituyen los Programas de Asistencia al Empleado (PAE), especialmente desarrollados en el ámbito de las adicciones.

Desde los años 1940, primero en Estados Unidos y luego en otros países (principalmente Europa y Latinoamérica) se han desarrollado PAEs para lograr un medio laboral saludable y seguro, proteger al trabajador, al empleador y a terceras personas de las consecuencias adversas del abuso de sustancias adictivas y de otros trastornos 
mentales. Surgen ante la realidad de que los problemas y presiones, ya sean de la vida personal o laboral, repercuten directamente en la salud y en el desempeño del empleado en sus dos esferas principales: personal y laboral.

Se trata de un servicio confidencial, proporcionado por consultoras o servicios externos o desde la propia organización, que provee al empleado de diversa información y atención. Algunos servicios ofertados se centran en el trabajador y otros se extienden a su ámbito familiar.

Según los diferentes objetivos y estructura de los PAE, los principales servicios ofertados son:

- Asesoría y tratamiento médico. Incluyendo salud mental, control del peso y nutrición, adicciones (tabaco, alcohol y otras drogas), y desarrollo de hábitos saludables (ejercicio físico por ejemplo).

- Asesoría y tratamiento psicológico. En salud mental y adicciones, situaciones de crisis, preparación para la jubilación, afrontamiento del estrés, etc.

- Asesoría e intervención jurídica (por ejemplo ante despidos y otras situaciones laborales o divorcios y otras situaciones personales).

Algunos estudios sobre las características y beneficios-costes de los PAE ${ }^{44-46}$, estiman que con la implantación de estos servicios se ahorran entre 3 y 15 dólares por cada dólar invertido en el Programa (en organizaciones como General Motors o United Airlines). La empresa de aviación Boeing, indica que desde que implantó el PAE en su organización ha ahorrado 5,1 millones de dólares por la reducción de las ausencias laborales, rotaciones y bajas médicas y Chevron, una compañía energética americana, reporta una mejora en el desempeño de un $50 \%$ en los empleados que han recibido apoyo por parte del PAE.

Se han diseñado varios programas de intervención en adicciones ampliamente utilizados $^{21,47}$, pero en general, todos los programas comparten dos componentes esenciales:

- El entrenamiento, con adecuada formación, información.

- Y una estrategia de "confrontación constructiva" sobre las variables del propio trabajador que participan del problema.

En España, dentro del ámbito sanitario, ha sido pionero el Programa de Atención al Médico Enfermo (PAIME), creado por los Colegios Médicos Profesionales y con la colaboración de la Organización Médica Colegial y el Programa de Atención Integral al Profesional Sanitario Enfermo (PAIPSE), para la atención a personal sanitario en general.

Finalmente, si hablamos de promoción de la salud mental, esto implicará la creación de condiciones individuales, sociales y medioambientales que habiliten el desarrollo óptimo, psicológico y psicofisiológico, a través del fortalecimiento de los factores protectores que reducen la aparición y la recurrencia de trastornos mentales ${ }^{48}$. Es decir, no centrarse solo en la enfermedad, en lo que no funciona, sino potenciar lo saludable, los recursos, tanto del individuo como del entorno laboral.

En la literatura sobre estos temas se han definido algunas orientaciones generales que pueden contribuir a definir políticas de promoción de la salud mental en el trabajo $^{49}$ :

1. El objetivo global de la promoción de la salud mental es que los trabajadores tengan un mayor grado de control sobre su salud y bienestar, así como sobre las condiciones de trabajo que inciden en aquéllas, mejorando a la vez el ajuste con su medio ambiente humano y material.

2. La promoción de la salud mental debe enfatizar los aspectos salutogénicos del trabajo y privilegiar la prevención primaria, en contraste con la detección de enfermedades y su tratamiento y rehabilitación. 
3. Las acciones de promoción deben estar dirigidas principalmente a los trabajadores sanos, incluyendo a aquéllos con síntomas inespecíficos de estrés, en todos los niveles de las organizaciones.

4. Más allá del estado de salud de los miembros individuales, las estrategias de promoción y prevención primaria deben dirigirse hacia el objetivo más amplio de lograr organizaciones saludables.

5. La promoción de la salud mental involucra cambios en valores, actitudes y comportamientos en los individuos y, por lo tanto, cambios en la cultura de la organización. Es requisito el compromiso de los líderes de la organización y la participación de los trabajadores en todos los niveles.

6. Las actividades de promoción de la salud mental pueden desarrollarse localmente, en cada empresa u organización individual y como un componente más de programas existentes o que se desee introducir.

7. La introducción de actividades de promoción de la salud mental, así como programas de prevención primaria en salud general, en empresas pequeñas y medianas implica dificultades adicionales y constituye un desafío. Puede facilitarse por medio de la información apropiada.

\section{CONCLUSIONES}

El estrés del trabajo se ha convertido en una de las principales causas de incapacidad laboral en los países desarrollados, al mediar en nuevas amenazas para la salud mental y como factor de riesgo de otras enfermedades como las cardiovasculares y los los accidentes de trabajo.

Según la revisión realizada, la relación entre salud mental y salud laboral es compleja y multifactorial. La relación entre los factores laborales y el trastorno mental puede darse de diferentes maneras:

- Coincidente temporal que el trabajador considera causa de su trastorno. Depende de procesos atribucionales y características de personalidad como la capacidad de introspección y el "locus de control". Una persona con poco "insight" y tendencia a hacer atribuciones estables y globales de atribución externa para los sucesos negativos, tenderá a atribuir dificultades propias a factores ajenos a él mismo. También patologías con sintomatología paranoide, por ejemplo, pueden focalizarse en personas o entornos laborales.

- Magnificador. El estresor revela y magnifica una alteración ya presente en el individuo (por una incidencia patológica en su estado cognitivo, emocional y/o conductual)

- Consecuencia. Una situación laboral, como por ejemplo la vivencia en el lugar de trabajo de una agresión por parte de un usuario, puede generar un nivel de estrés patológico y un trastorno mental consecuencia del mismo (TEPT por ejemplo). Para poder hacer de forma adecuada esta diferenciaciones esencial el análisis de la secuencia de los acontecimientos.

- Precipitante o desencadenante de un trastorno mental para el cual está predispuesto genéticamente o por la interacción genético-ambiental desde el nacimiento.

La prevención primaria, secundaria y terciaria, junto a medidas de promoción de la salud en los lugares de trabajo, permitirán el desarrollo de la salud mental laboral mediante el diagnóstico precoz y el tratamiento eficaz de los distintos trastornos y sus aspectos laborales relacionados. Todo ello redundará en el logro de los siguientes objetivos:

a) La mejora continua de la calidad de vida laboral:

- Promoviendo programas de salud mental en el ámbito de la salud laboral con un modelo de trabajo interdisciplinario e integrador de las distintas actuaciones 
sanitarias, preventivas y de promoción de la salud con integración de la salud mental en la salud general.

- Establecer protocolos de derivación, coordinación y reincorporación laboral de los trabajadores con trastorno mental.

- Desarrollar actividades formativas compartidas entre salud mental y salud laboral con intercambio bidireccional de conocimientos teóricos y destrezas prácticas a través de sesiones de interconsulta de casos, sesiones clínicas, teóricas, grupos tipo Balint, etc.

- Colaboración mutua en los aspectos relacionados con la investigación de los trastornos mentales en el ámbito laboral.

b) La reducción de los costes de la enfermedad y una gestión eficiente de los recursos.

Para lo que será importante priorizar el control, la regulación y la prevención del riesgo. Intervenciones como las realizadas por los Programas de Asistencia al Empleado, han demostrado su eficacia y rentabilidad en este sentido.

Trabajar sobre la relación entre los problemas laborales y la salud mental, exigirá la colaboración entre los Servicios de Recursos Humanos, Prevención de Riesgos Laborales, Salud Mental, Inspección, Atención Primaria, etc. A su vez, será necesario evitar el uso indiscriminado e instrumental del abordaje psiquiátrico o psicológico por la búsqueda de un beneficio secundario. Sirva de ejemplo la búsqueda de informes psiquiátricos con la finalidad de un cambio de puesto de trabajo pero sin la intención de buscar la atención psíquica del problema. La posible intervención en el ámbito psíquico tendrá siempre el objetivo de buscar la salud y bienestar del individuo y si el problema está relacionado con el entorno laboral, la prevención "en origen", tal y como estipula la Ley de Prevención de Riesgos Laborales, será una pieza clave para su resolución.

No quisiéramos terminar este trabajo sin recordar que la salud mental implica más que la ausencia de un trastorno mental. Las personas psíquicamente sanas presentan ante algunas circunstancias de conflictos personales, sociales o laborales, un rango de conductas, emociones y cogniciones, que pueden ser poco adaptativas y/o funcionales para su propia salud y para la resolución de dicho problema sin que por ello se trate de un trastorno mental.

\section{REFERENCIAS BIBLIOGRÁFICAS}

1. Regier DA, Boyd JH, Burke JD y cols. One month prevalence of mental disorders in the United States. Based on five epidemiologic catchment area sites. Arch Gen Psychiatry 1988; 45: 977-86.

2. Regier DA, Narrow WE, Rae DS y col. The de facto U.S. mental and addictive disorders service system:Epidemiologic Catchment Area prospective one-year prevalence rates of disorders and services. Arch Gen Psychiatry 1993; 50: 85-94.

3. Otero FJ. Trastornos psiquiátricos y Atención Primaria. Barcelona: Doyma S.A.; 1995.

4. Martín Zurro A. Salud Mental y Atención Primaria. Barcelona: Doyma S.A.; 1995.

5. DSM-IV-TR. Manual diagnóstico y estadístico de los trastornos mentales. Texto Revisado. Barcelona: Masson, 2002.

6. Palao DJ, Márquez M, Jódar I. Guía Psiquiátrica en Atención Primaria. Madrid: SmithKline Beecham; 1996.

7. Steptoe A, Appels A. Stress, Personal Control and Health. Chichester: John Wiley \& Sons; 1989.

8. Rutter DR, Quine L. Social Psychology and Health: European Perspectives. Avebury. Aldershot; 1994.

9. Steckler T, Kalin NH, Reul JM. Handbook of Stress and the Brain. Integrative and Clinical Aspects. Amsterdam: Elsevier; 2005.

10. Ley de Prevención de Riesgos Laborales 31/1995 de 8 de noviembre y actualización mediante Ley $25 / 2009$ de 22 de diciembre.

11. Mingote JC. Satisfacción, estrés laboral y calidad de vida del médico. Tesis Doctoral. Universidad Autónoma de Madrid; 1995. 
12. Stanfeld SA. Work, personality and mental heath. Br J Psychiatry 2002; 181: 96-98.

13. Fundación Galatea, OMC. La salud del MIR. Guía para los Servicios de Prevención de Riesgos Laborales. Disponible en: http://www.fgalatea.org/Upload/Documents/6/66.pdf

14. Phisycian Health Services, Inc. Massachusetts. Disponible en: http://www.massmed.org/Content/ NavigationMenu6/HelpingYourselforaColleague/Signs_of_Concern_PH.htm

15. Goldberg DP, Huxley PJ. Mental illness in the community: The pathway to psychiatric care. Londres: Tavistock; 1980.

16. Muñoz PE. Epidemiología de los trastornos mentales. En: Vallejo, Leal (Dir). Tratado de Psiquiatría Vol I. Barcelona: Ars Médica; 2005.

17. Trucco M. El estrés y la salud mental en el trabajo: Documento de Trabajo de la Asociación Chilena de Seguridad. Ciencia \& Trabajo 2004; 14: 185-88.

18. Gimeno D, Benavides FG, Benach J, Jarque S, Cambra S, Devesa J. Trastornos afectivos en la población laboral: ¿un problema emergente en salud laboral?. Med Clin (Barc) 2001; 116: 493-95.

19. Mingote JC, Gálvez M, Del Pino P, Gutiérrez MD. El paciente que padece un trastorno depresivo en el trabajo. Med Seg Tr 2009; 55(214): 41-43.

20. Córdoba JJ, Fuentes A, Ruiz C. Revisión bibliográfica sobre características sociodemográficas y repercusiones de la depresión en el trabajador. Med Seg Tr 2011; 57(223): 186-96.

21. Cook RF, Back AS, Trudeau $\mathfrak{j}$, McPherson TL. Integrating substance abuse prevention into health promotion programs in the workplace: A social cognitive intervention targeting the mainstream user. En: Bennett JB, Lehman EK (Eds), Preventing workplace substance abuse: Beyond drug-testing to wellness. Washington, DC: American Pycological Association; 2003.

22. Snow DL, Swan SC, Wilton L. A workplace coping-skills intervention to prevent alcohol abuse. En Bennett JB, Lehman EK (Eds), Preventing workplace substance abuse: Beyond drug-testing to wellness. Washington, DC: American Pycological Association; 2003.

23. Mezzich JE, Salloum IM. Towards innovative international classification and diagnosis systems. ICD-11 and person-centered integrative diagnosis. Acta Psychiatr Scand 2007; 116:1-5.

24. Frances A, Ross R. DSM-IV-TR. Estudio de Casos: Guía clínica para el diagnóstico diferencial. Masson; 2002.

25. Brown GW, Harris TO. The Bedford College Life-Events and Difficulty Schedule: Directory of contextual threat ratings of events. Londres: Bedford College; 1989.

26. Goldberg D. Manual del General Health Questionnaire. Windsor: NFER Publishing, 1978.

27. Jacobsen BK, Hasvold T, Hoyer G. Hansen V. The general health questionnaire. How many items are really necessary in population surveys. Psychol Med 1995;25(5):957-61.

28. Hemert van, Heijer M den. Vorstenbosh M, Bolk JH. Detecting psychiatric disorders in medical practice using the general health questionnaire. Why do cutt - off scores vary. Psychol Med 1995;25(1):165-70.

29. Goldberg DP, Hiller VF. A scaled version of the general health questionnaire. Psychol Med 1979;(1): $139-45$.

30. Derogatis LR, Lipman RS, Covi L. SCL-90: An outpatient psychiatric rating scale preliminary report. Psychopharmacol Bull 1973; 9: 13-28.

31. Davison MK, Bershadsky B, Bieber J, Silversmith D, Maruish ME, Kane RL. Development of a brief, multidimensional, selfreport instrument for treatment outcomes assessment in psychiatric settings: Preliminary findings. Assessment 1997; 4, 259-276.

32. Sandin B, Valiente RM, Chorot P, Santed MA, Lostao L. SA-45: forma abreviada del SCL-90. Psicothema 2008; 20(2): 290-96.

33. Zúñiga A. Estudio de la casuística en un Centro de Salud Mental Comunitario: gravedad y utilización de servicios. Tesis doctoral. Departamento de Psiquiatría y Medicina Legal, Universidad de Barcelona 2008.

34. Mingote JC, del Pino P, Gálvez M, Gutiérrez MD, Sánchez R. Utilidad preventiva del constructo "trastorno mental grave" en el ámbito sociosanitario. Med Seg Tr 2010; 56(221): 306-322.

35. Mingote JC, del Pino P, Huidobro A, Gutiérrez MD, de Miguel I, Gálvez M. El paciente que padece un trastorno psicótico en el trabajo: diagnóstico y tratamiento. Med Seg Tr 2007; Vol LIII(208): 29-52.

36. Ciurana R, Tizón JL. Prevención de los trastornos de la salud mental desde la Atención Primaria de Salud. Propuesta de Priorización en la prevención de los trastornos de la salud mental. En: Red de Centros Investigadores de la SEMFYC Ed., Programa de Actividades Preventivas y de Promoción de la Salud en Atención Primaria, Libro de la V Reunión Anual. Barcelona: Sociedad Española de Medicina de Familia y Comunitaria; 1993. 
37. Gálvez M, Mingote JC, Moreno-Jiménez B. El paciente que padece un trastorno de personalidad en el trabajo. Med Segur Trab 2010, 56(220):226-47.

38. Othmer E, Othmer SC. La entrevista clínica, tomos I y II. Barcelona: Masson; 1996.

39. Furnham A. The Psychology of Behaviour at Work. The individual in the organization. Hove, U.K: Psychology Press Taylor and Francis Group; 1997.

40. Peiró JM, Prieto F, Roe RA. La aproximación psicológica al trabajo en un entorno laboral cambiante. En: Peiró JM, Prieto F, (Eds.). Tratado de Psicología del Trabajo. Madrid: Síntesis; 1996. p. 15-36.

41. Benavides FG, Ruiz-Frutos C, García AM. Salud Laboral. Conceptos y técnicas para la prevención de riesgos laborales ( $2^{\mathrm{a}}$ ed.) Barcelona: Masson; 2000.

42. García M, de la Casa S, Molina C. Regulación de los riesgos psicosociales en los ambientes de Trabajo: panorama comparado de modelos y experiencias en Europa y América. Albacete: Ed. Bomarzo; 2011.

43. Rau R, Morling K, Rösler U. Is there a relationship between major depression and both objectively assessed and perceived demands and control?. Work \& Stress 2010, 24 (1): 88-106.

44. Federal Register USA. Department of Health and Human Services. Mandatory Guidelines for Federal Workplace. Drug Testing Programs. USA; 1988.

45. Coscatl. Programa de Asistencia al Empleado. Disponible en: http://www.coscatl.com/articulos/ programa-asistencia-empleado.htm

46. Recursos Nacional Canadiense de Seguridad y Salud Ocupacional. Programas de Asistencia al Empleado. Disponible en: http://www.ccsso.ca/oshanswers/hsprograms/eap.html

47. Bray JW, Galvin DM, Cluff LA. (eds.) Young Adults in the workplace: A Multisite Initiiative of Substance Use Prevention Programs. RTI Press publication. Disponible en: http://www.rti.org/rtipress

48. Hosma C, Jane-Llopis E, Saxena S (Eds.). Prevention of mental disorders: Effective Interventions and Policy Options. Oxford University Press; 2005.

49. Trucco M. Promoción de la salud mental en el ámbito laboral. Informe a la OPS. Washington D.C.; 1998.

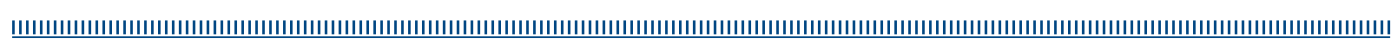

